\title{
Tests of Lorentz and CPT violation with neutrinos
}

\author{
Teppei Katori * \\ Laboratory for Nuclear Science, \\ Massachusetts Institute of Technology, \\ Cambridge, USA \\ E-mail: katori@mit.edu
}

Lorentz violation has been shown to occur in the Planck scale theories. Although the three active massive neutrino framework with the Standard Model (SM), so-called the neutrino Standard Model ( $v \mathrm{SM})$, is successful, series of signals not understood within the $v \mathrm{SM}$ suggest neutrino physics may be the first place to see the physics beyond the SM, such as Lorentz violation. Especially, neutrino oscillations are the natural interferometer and they are sensitive to the Lorentz violation with comparable sensitivity with precise optical experiments.

The LSND oscillation signal was analyzed under the Standard Model Extension (SME) framework, and it was found that the oscillation data was consistent with no Lorentz violation, but data cannot reject Lorentz violation hypothesis. By assuming LSND signal was due to the Lorentz violation, a global phenomenological model was made to describe all known oscillation data including the LSND signal. The model also predicted the signal for MiniBooNE at the low energy region.

Later, MiniBooNE announced an event excess at the low energy region. However, the oscillation candidate signals from MiniBooNE were consistent with no Lorentz violation. The limit obtained by MiniBooNE and MINOS on the $e-\mu$ sector reject the simple scenario to explain LSND signal with Lorentz violation.

Meantime, MINOS and IceCube set tight limits on the $\mu-\tau$ sector Lorentz violation. The last untested channel, the $e-\tau$ Lorentz violating mixing, was tested using reactor disappearance data from Double Chooz. However, Double Chooz data was consistent with flat, and sidereal time dependent Lorentz violation hypothesis is rejected. Combinations of all oscillation data from LSND, MiniBooNE, MINOS, IceCube, and Double Chooz provide very tight constraint for a possible Lorentz violation in the neutrino sector in terrestrial level.

36th International Conference on High Energy Physics,

July 4-11, 2012

Melbourne, Australia

${ }^{*}$ On behalf of LSND, MiniBooNE, and Double Chooz collaborations 

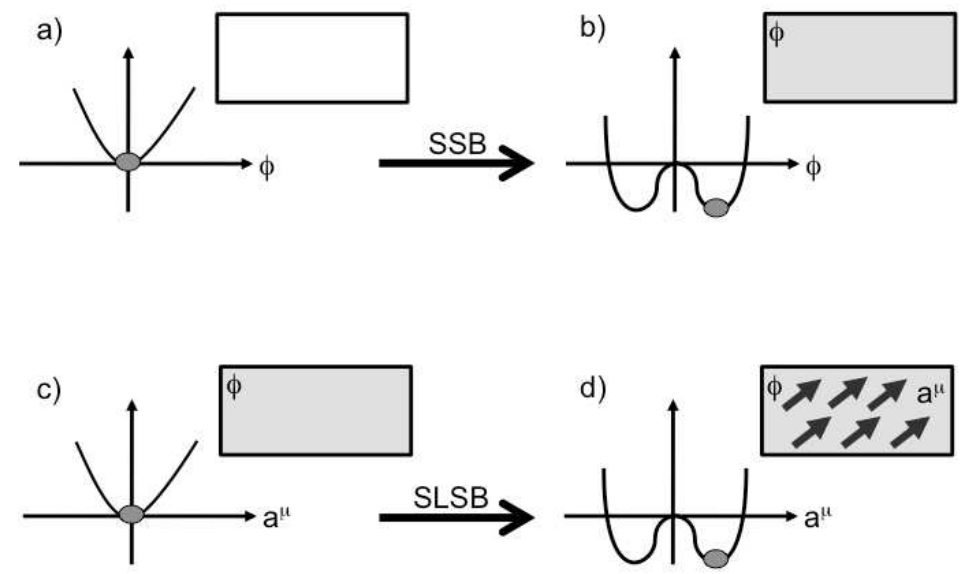

Figure 1: An illustration of spontaneous symmetry breaking (SSB). Figure is taken from [1].

\section{Introduction}

\subsection{Spontaneous Lorentz Symmetry Breaking (SLSB)}

Lorentz violation is a predicted phenomenon from the Planck scale physics. Especially if Lorentz violation were made by the spontaneous process, quantum field theory and general relativity would require no modifications. Figure 1 shows a cartoon of this situation. When the universe is hot, the scalar field preserves the perfect symmetry (Fig. 1a). But once it gets cold, there is a chance that the potential of the scalar field shifts to the "Mexican hat" potential (Fig. 1b), and nonzero field value is more stable, i.e. the vacuum acquires the vacuum expectation value of this scalar field. If the scalar field has any quantum numbers, say SU(2) charge, such quantum numbers are not preserved in the vacuum, namely chirality is not conserved for massive particles in the SM vacuum. This is the spontaneous symmetry breaking (SSB) in the SM, and this year is the great year for this mechanism since the strong candidate of Higgs particle is discovered, and presented at this ICHEP2012 conference!

This process can be extended to any fields with Lorentz indices beyond the scalar field. For simplicity I discuss only the vector field. When the universe is hot, a vector field keeps the perfect symmetry (Fig. 1c). But again, when universe gets cold, the vector field could generate a nonzero vacuum expectation value (Fig 1d). This is the situation of the spontaneous Lorentz symmetry violation (SLSB) [2], and Lorentz symmetry is spontaneously broken. In this case, the universe is filled with the background vector field represented by the arrows. If the SM particles couple with those fields, their physics depend on the orientation of the arrows or direction.

Since such background fields of the universe are fixed in the space, presence of such coupling implies the direction dependent physics. In particular, rotation of the Earth (period $86164.1 \mathrm{sec}$ ) causes sidereal time dependent physics for terrestrial measurements. Therefore, sidereal time de- 


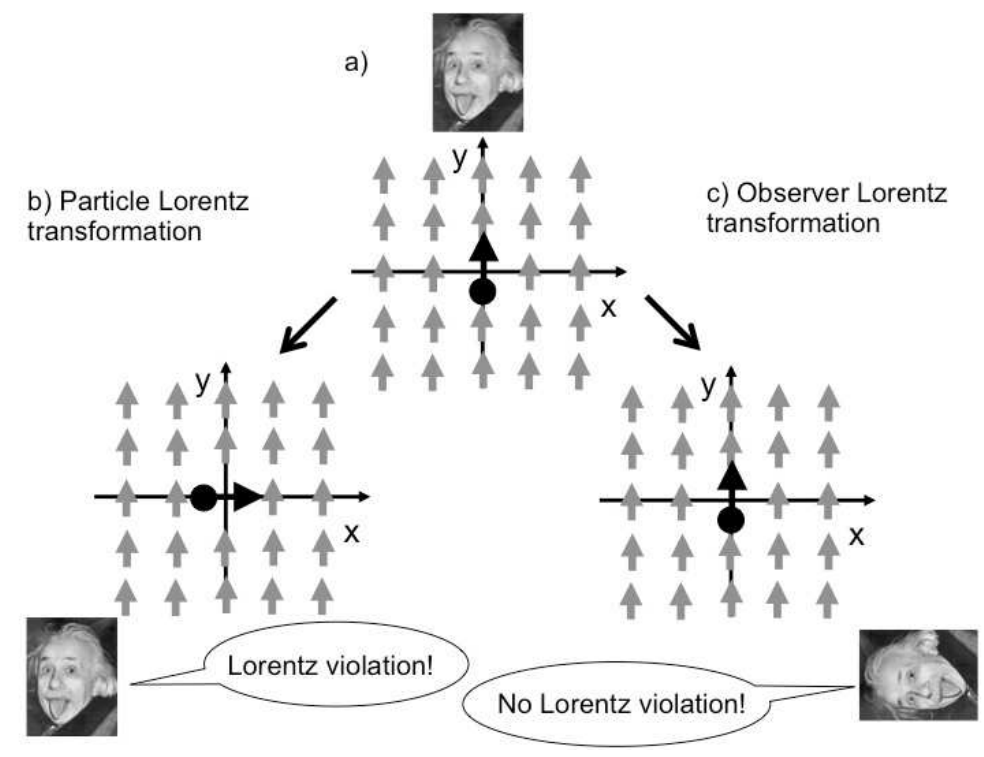

Figure 2: An illustration of the Particle Lorentz transformation and the Observer Lorentz transformation. Figure is taken from [1].

pendence of physics observables is the smoking gun of the Lorentz violation.

\subsection{Particle and Observer Lorentz transformation}

Lorentz violation is more precisely the violation of the Particle Lorentz transformation. This situation is described in Figure 2. In Fig. 2a, a SM particle is moving upward in two-dimensional space where the hypothetical background vector field saturates the space (depicted by arrows), and Einstein represents a local observer.

The change in motion of a SM particle within a fixed coordinate system is described by Particle Lorentz transformation (Fig. 2b). Since the background field is unchanged, as a consequence, a coupling between the SM particle and vector field is not preserved. In general, Lorentz violation means Particle Lorentz violation.

On the other hand, local observer's inverse coordinate change can also generate change in the motion of a SM particle (Fig. 2c). In the theory without Lorentz violation, this Observer Lorentz transformation coincides with the Particle Lorentz transformation. However, as you see, this corresponds to mere coordinate transformation and the coupling of the SM particle and the background field is preserved. Therefore, Lorentz-violating effect is conserved by the coordinate transformation, and it can be studied in any frames or coordinates.

\subsection{Test of Lorentz violation with neutrino oscillations}

Lorentz violation is realized as a coupling of SM particles and the background field of the universe. Although the Lorentz-violating phenomenon is coordinate independent, we need to choose a coordinate system so that we can report measurements of the coefficients of such fields [3]. Figure 3 shows our scheme. First, the motion of the Earth is described in the Sun-centered coordinate system (Fig. 3a). This coordinate system provides the bases for the Lorentz violating fields to specify the 

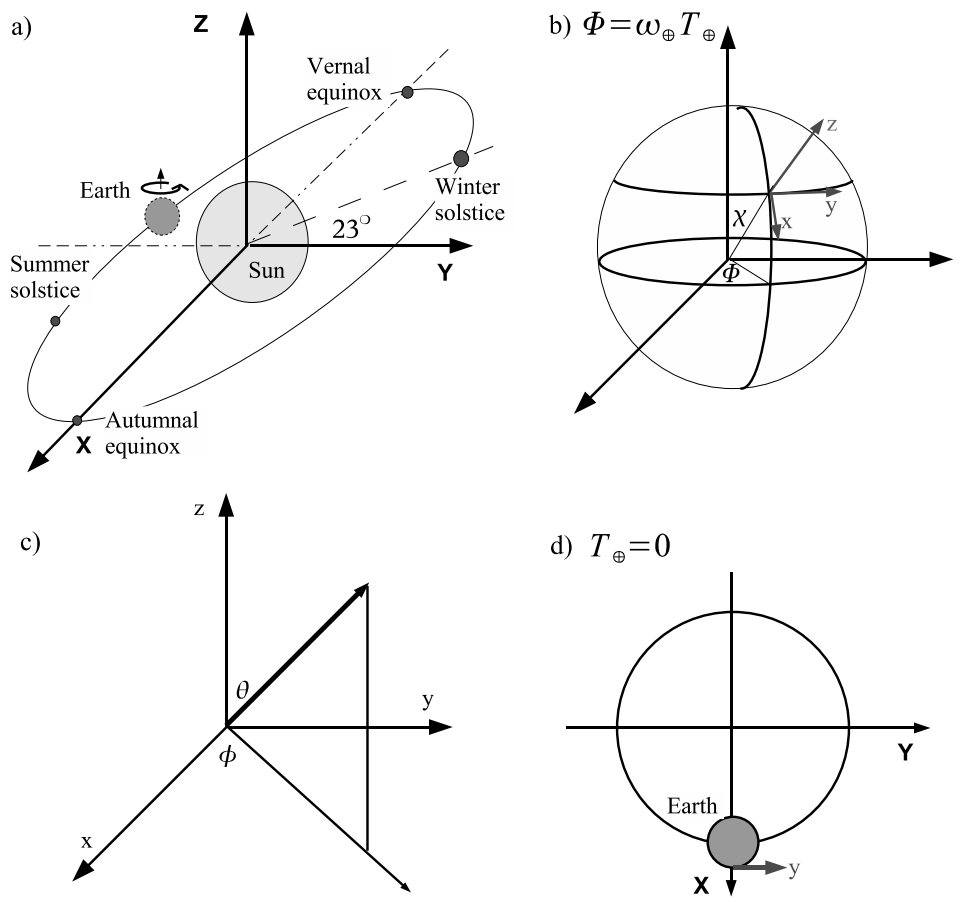

d) $T_{\oplus}=0$

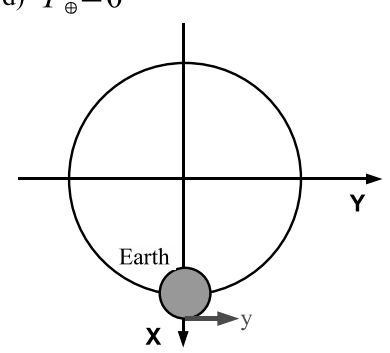

Figure 3: The coordinate system used by this analysis, the Sun-centered coordinates (a), the Earth-centered coordinates (b), and the local polar coordinate system (c). The time zero is defined when the experiment site is at midnight near the autumnal equinox, in other words, when the large "Y" and small " $y$ " axes almost align (d). Figure is taken from [5].

coefficients. Then the location of the experimental site is specified in the Earth-centered coordinate system (Fig. 3b). Finally, the direction of SM particles (i.e., direction of the beam) is described in the local polar coordinate system (Fig. 3c).

The Standard Model Extension (SME) [4] is constructed as a general framework to analyze the data to search possible Lorentz violation. In the SME, Lorentz violating interactions are described by the perturbative terms in the Lagrangian, on top of the SM terms. We are particularly interested in the test of Lorentz violation using neutrino oscillations. Since neutrino oscillation is a natural interferometer, small couplings of neutrinos with Lorentz violating fields could cause phase shifts and could result in neutrino oscillations. The sensitivity of neutrino oscillations to Lorentz violation is comparable with precise optical experiments. To analyze the neutrino data we use the neutrino sector SME [6] ${ }^{1}$,

$$
\begin{aligned}
\mathscr{L} & =\frac{1}{2} i \bar{\psi}_{A} \Gamma_{A B}^{\mu} \stackrel{\leftrightarrow}{D_{\mu}} \psi_{B}-\bar{\psi}_{A} M_{A B} \psi_{B}+\text { h.c. } \\
\Gamma_{A B}^{v} & \equiv \gamma^{v} \delta_{A B}+c_{A B}^{\mu v} \gamma_{\mu}+d_{A B}^{\mu v} \gamma_{5} \gamma_{\mu}+e_{A B}^{v}+i f_{A B}^{v} \gamma_{5}+\frac{1}{2} g_{A B}^{\lambda \mu v} \sigma_{\lambda \mu}
\end{aligned}
$$

\footnotetext{
${ }^{1}$ In this article, we limit ourselves within the renormalizable SME only. However, the results in this article can also set the limits on the nonrenormalizable SME coefficients [7].
} 


$$
M_{A B} \equiv m_{A B}+i m_{5 A B} \gamma_{5}+a_{A B}^{\mu} \gamma_{\mu}+b_{A B}^{\mu}+\frac{1}{2} H_{A B}^{\mu v} \sigma_{\mu \nu} .
$$

Here, the $A B$ subscripts represent flavor space. The first term of Eq. 1.2 and the first and the second terms of Eq. 1.3 are the only nonzero terms in the SM. The rest of the terms are from the SME. These SME coefficients can be classified into two groups: $e_{A B}^{\mu}, f_{A B}^{\mu}, g_{A B}^{\mu \nu \lambda}, a_{A B}^{\mu}$, and $b_{A B}^{\mu}$ are CPT-odd SME coefficients, and $c_{A B}^{\mu v}, d_{A B}^{\mu \nu}$, and $H_{A B}^{\mu v}$ are CPT-even SME coefficients.

In this way, physical observables can be written down including Lorentz violation. By assuming the baseline is short enough for the oscillation length the neutrino oscillation probability $\alpha \rightarrow \beta$ can be written as follows $[8]^{2}$,

$$
\begin{aligned}
P_{\alpha \rightarrow \beta} \simeq & \frac{L^{2}}{(\hbar c)^{2}} \mid(\mathscr{C})_{\alpha \beta}+\left(\mathscr{A}_{S}\right)_{\alpha \beta} \sin \omega_{\oplus} T_{\oplus}+\left(\mathscr{A}_{c}\right)_{\alpha \beta} \cos \omega_{\oplus} T_{\oplus} \\
& +\left(\mathscr{B}_{s}\right)_{\alpha \beta} \sin 2 \omega_{\oplus} T_{\oplus}+\left.\left(\mathscr{B}_{c}\right)_{\alpha \beta} \cos 2 \omega_{\oplus} T_{\oplus}\right|^{2}
\end{aligned}
$$

Here, $\omega_{\oplus}$ is the sidereal time angular frequency $\left(\omega_{\oplus}=\frac{2 \pi}{86164.1} \mathrm{rad} / \mathrm{s}\right)$. The neutrino oscillation probability is described by the function of the sidereal time $T_{\oplus}$ with five amplitudes. $(\mathscr{C})_{\alpha \beta}$ is the sidereal time independent amplitude, and $\left(\mathscr{A}_{S}\right)_{\alpha \beta},\left(\mathscr{A}_{c}\right)_{\alpha \beta},\left(\mathscr{B}_{S}\right)_{\alpha \beta}$, and $\left(\mathscr{B}_{c}\right)_{\alpha \beta}$ are the sidereal time dependent amplitudes. Therefore, an analysis of Lorentz and CPT violation in neutrino oscillation data involves fitting the data with Eq. 1.4 to find nonzero sidereal time dependent amplitudes. These amplitudes are written by a combination of the SME coefficients, and the explicit expressions are given at elsewhere [8].

\section{Lorentz violation analysis on LSND experiment}

LSND is an appearance neutrino oscillation experiment at Los Alamos. A low energy $\bar{v}_{\mu}$ beam $(\sim 40 \mathrm{MeV})$ was made by pion decay-at-rest. The detector was located $\sim 30 \mathrm{~m}$ away from the target. LSND observed the excess of $\bar{v}_{e}$ candidate events from the $\bar{v}_{\mu}$ beam [10], and this result is not understood within the $v \mathrm{SM}$. Thus, LSND signal may be the signal of new physics, such as sterile neutrino oscillations. However, it may be the first signal of Lorentz violation. If this is the case, interference pattern, i.e., the number of the oscillation candidates, would show the sidereal time dependence.

We analyzed the LSND oscillation candidate data with a function of the sidereal time [5]. We fit Eq. 1.4 to find the best parameter set by an unbinned likelihood method. Fig. 4 shows the result. The data is consistent with a flat hypothesis (=no sidereal time dependence), however small Lorentz violation (=sidereal time dependent solution) is not rejected. If that is the case, LSND oscillation candidate is explained by order $10^{-17}$ CPT-odd Lorentz violation and/or order $10^{-19} \mathrm{GeV}$ CPTeven Lorentz violation [11].

\section{Global neutrino oscillation model with Lorentz violation}

Although LSND signal could be described by nonzero Lorentz violating parameters, naively such new parameters would be forbidden by other oscillation experiment data. We examined the

\footnotetext{
${ }^{2}$ If this is not the case, Lorentz violation can be studied as perturbations of standard oscillations [9]. Undermentioned MINOS far detector and IceCube analyses are based on this scheme.
} 


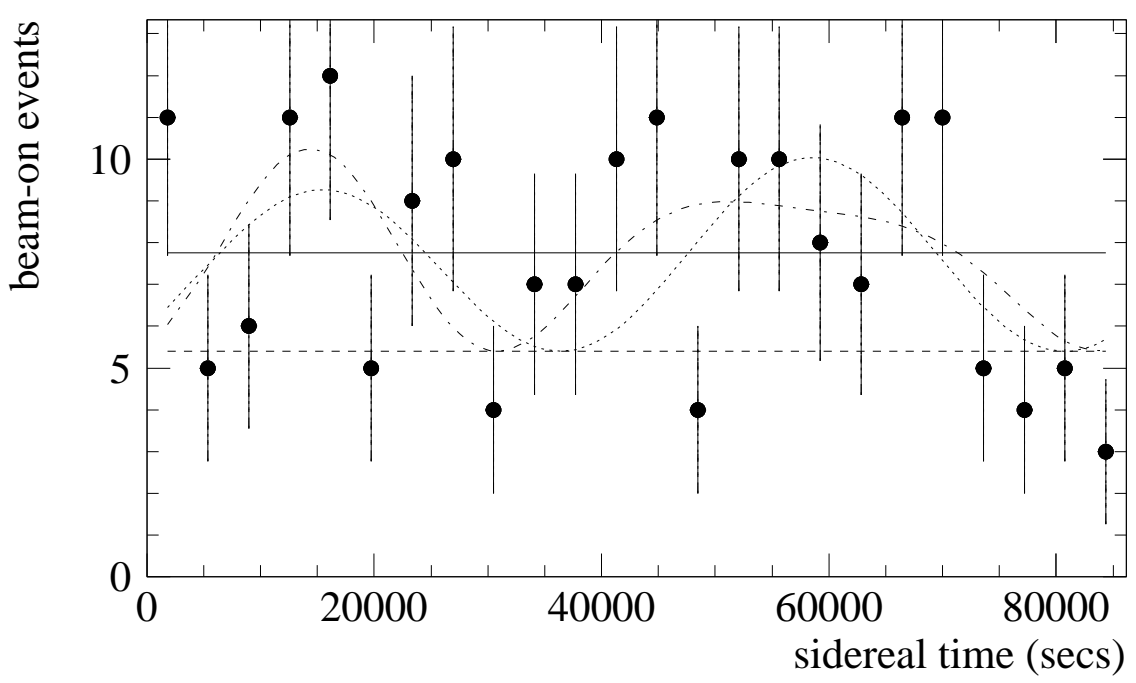

Figure 4: Sidereal time distribution of the LSND oscillation candidate events (marker). Data is fit with oscillation models including sidereal time dependence (dotted and dot-dashed curves) and flat hypothesis (solid line). The background is assumed to be flat (dashed line). Figure is taken from [5].

possible phenomenological models to describe the world oscillation data including LSND. The "tandem" model was made in such concept [12] (as an extension of the "bicycle" model [13]). The tandem model satisfies all requirements as an alternative oscillation model. One of the very attractive features of this model is that it only uses three parameters to describe four oscillation signals; solar, atmospheric, KamLAND, and LSND signals. In 2006, the $v$ SM had four parameters (two mass differences and two mixing angles), so the tandem model was a more economical phenomenological model than the $v$ SM. Fig. 5 shows our prediction on the short baseline experiments. It reproduces a $\sim 0.1 \%$ level oscillation signal at LSND. On the other hand, the signal at KARMEN is smaller to be consistent with the observation. It also predicted an oscillation signal at the low energy region of MiniBooNE, both neutrinos and antineutrinos.

Later more advanced global oscillation models based on Lorentz violation had been proposed [14], but all of these models failed to reproduce recent reactor neutrino results [15], which is another great discovery of this year, and it is also being presented at this ICHEP2012 conference!

\section{Lorentz violation analysis on MiniBooNE experiment}

\subsection{MiniBooNE experiment}

MiniBooNE is the neutrino and antineutrino appearance experiment designed to confirm or reject LSND signals under the two massive neutrino oscillation hypothesis. The neutrino (antineutrino) beams are created by the Booster Neutrino Beamline (BNB) [16]. The $8 \mathrm{GeV}$ proton beam is extracted from the Fermilab Booster, and it is sent to the target where the collision with the target makes shower of mesons. The magnetic focusing horn surrounding the target selects either positive mesons or negative mesons and their decay-in-flight make $\sim 800 \mathrm{MeV} v_{\mu}$ or $\sim 600 \mathrm{MeV} \bar{v}_{\mu}$ beam. 


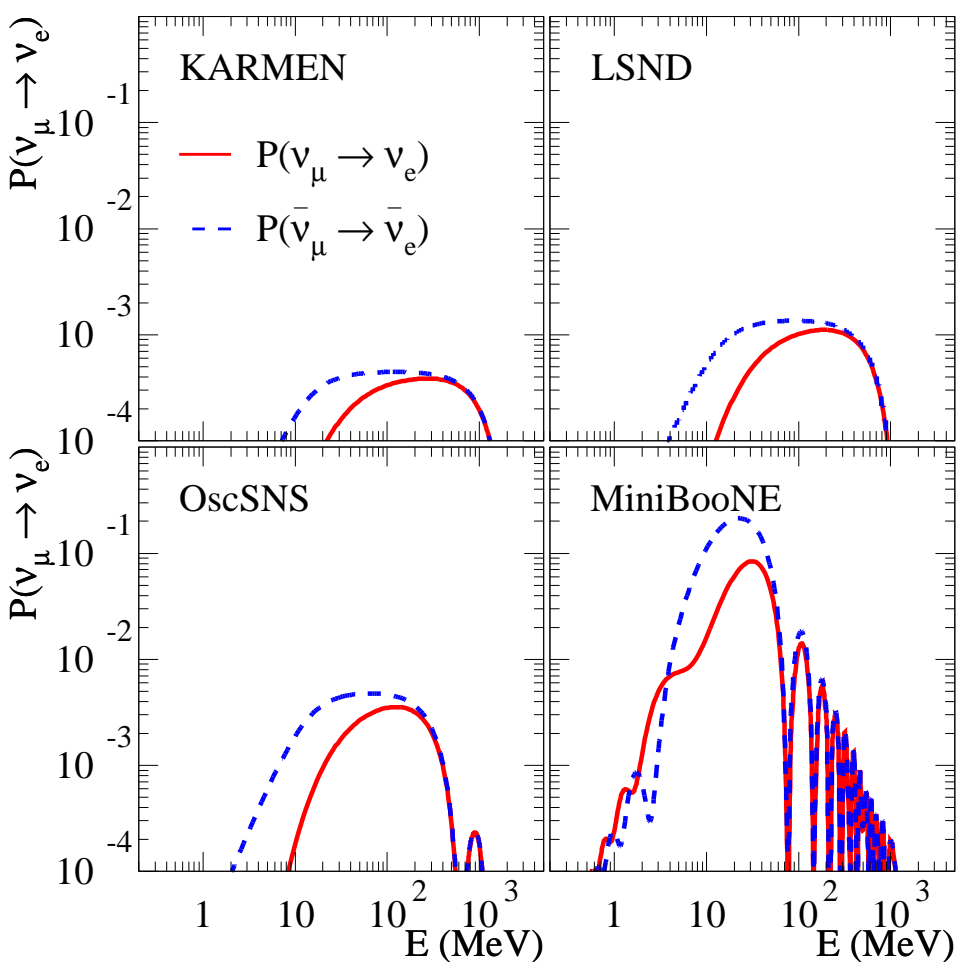

Figure 5: Oscillation probabilities as a function of energy for neutrino (solid line) and antineutrino (dashed lines). Figure is taken from [12].

The MiniBooNE detector is located $541 \mathrm{~m}$ away from the target [17]. It is a $12.2 \mathrm{~m}$ diameter spherical Cherenkov detector, filled with the mineral oil, and lined with 1,280 8-inch PMTs on the wall to observe the Cherenkov radiation from the charged particles.

The time and charge information of the PMTs from the charged particles are used to reconstruct charged particle momentum and particle type [18]. By assuming interaction is charged current quasielastic $(\mathrm{CCQE})$ and the target nucleon is at rest, the neutrino energy is reconstructed $(\mathrm{QE}$ assumption) [19]. It is vital to be able to reconstruct the neutrino energy for the neutrino oscillation physics.

\subsection{MiniBooNE oscillation analysis results}

The signature of the $v_{\mu} \rightarrow v_{e}\left(\bar{v}_{\mu} \rightarrow \bar{v}_{e}\right)$ oscillation is the single, isolated electron-like Cherenkov ring produced by the $\mathrm{CCQE}$ interaction.

$$
\begin{aligned}
& v_{\mu} \stackrel{\text { oscillation }}{\longrightarrow} v_{e}+n \rightarrow e^{-}+p, \\
& \bar{v}_{\mu} \stackrel{\text { oscillation }}{\longrightarrow} \bar{v}_{e}+p \rightarrow e^{+}+n .
\end{aligned}
$$

The cuts are designed to select such events. Both neutrino and anti-neutrino mode observed the event excesses. For the neutrino mode, MiniBooNE observed the event excess only in the low 


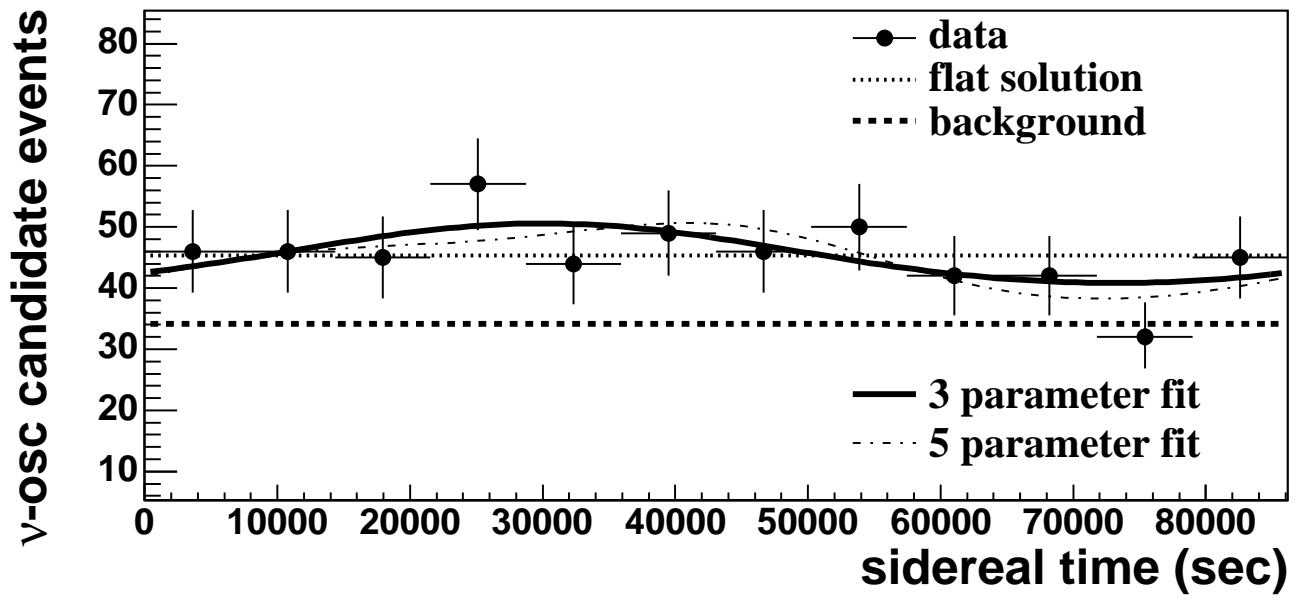

Figure 6: The fit results for the MiniBooNE neutrino mode low energy region. The plot shows the curves corresponding to the flat solution (dotted line), sidereal time dependent fits (solid and dash-dotted curves), together with binned data (solid marker). Here the fitted background is shown as a dashed line. Figure is taken from [23].

energy region [20]. The observed excesses cannot be described by the $v \mathrm{SM}$, so it may be the signal of new physics, such as Lorentz violation. On the other hand, for the antineutrino mode, the event excess is seen in the entire energy region [21]. Again, the observed excesses cannot be described by the $v \mathrm{SM}^{3}$. Since CPT violation naturally arises within Lorentz violation, this different patterns of excesses between neutrino mode and antineutrino mode is natural if it were caused by Lorentz violation. Therefore it is very interesting to take a look at their sidereal time distributions to find a possible Lorentz violation.

\subsection{MiniBooNE Lorentz violation analysis results}

We fit Eq. 1.4 to MiniBooNE neutrino mode low energy $v_{e}$ candidate excess and antineutrino model excess events. Figure 6 shows the neutrino mode low energy region fit result. As you see, data is quite consistent with a flat hypothesis. We constructed a fake data set without signal (=flat hypothesis) to evaluate the compatibility with a flat solution over the fit result by the $\Delta \chi^{2}$ method. It turns out data is compatible with a flat solution over a $26.9 \%$, and it concludes $v_{e}$ candidate data are consistent with no sidereal time dependence.

Figure 7 shows the antineutrino mode fit result. The fit result is more interesting here because the fit favors a sidereal time dependent solution. We again constructed a fake data set to find the significance of this solution, and it turns out that the compatibility with a flat solution is now only $3.0 \%$. Although this is interesting, the significance is not high enough to claim the discovery.

Since we didn't find the Lorentz violation, we set limits to fit parameters (=sidereal time dependent and independent amplitudes) [23]. From these limits one can extrapolate the limits to

\footnotetext{
${ }^{3}$ This analysis was done when only the half of the all antineutrino data set was available. Recently published full antineutrino mode data shows a somewhat different shape of the excess events [22].
} 


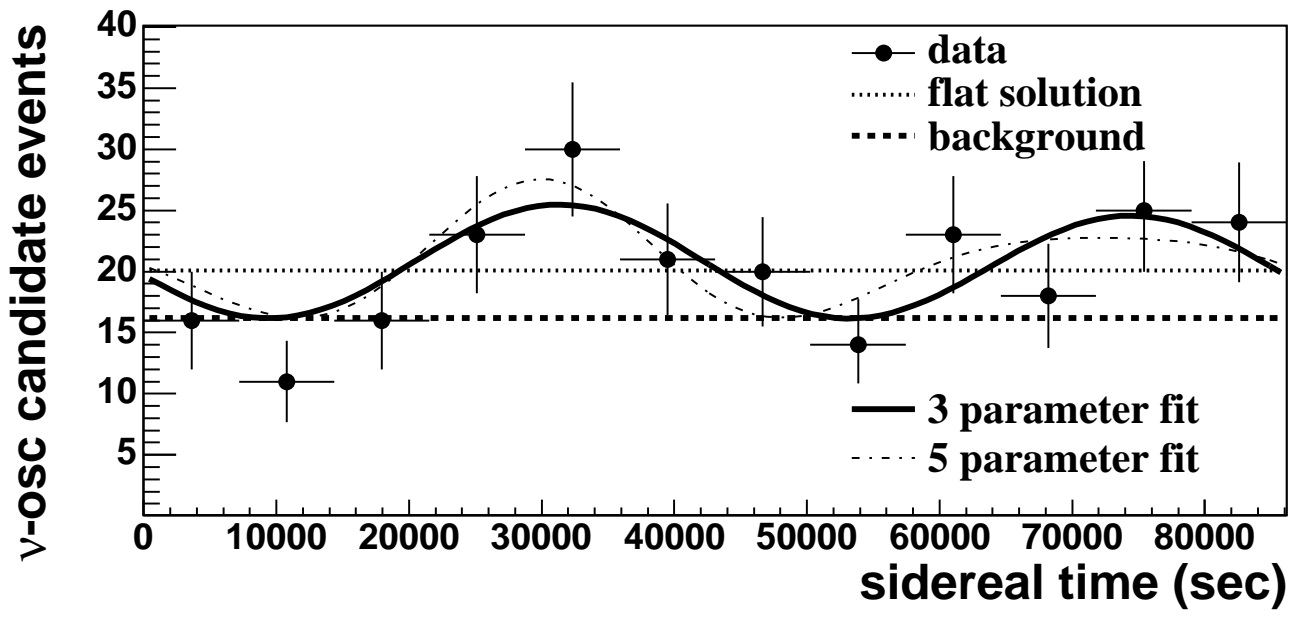

Figure 7: The fit results for the MiniBooNE antineutrino mode data. Notations are the same as previous figure. Figure is taken from [23].

the SME coefficients [1]. It turns out these limits indeed exclude the SME coefficients needed to explain the LSND signal. Therefore, there is no simple scenario to explain the LSND signal by Lorentz violation.

\section{Lorentz violation analysis on Double Chooz experiment}

From the MiniBooNE data analysis, we set limits on the $e-\mu$ oscillation channel SME coefficients. MINOS near detector analysis [24] also sets severe limits to some of these coefficients. Meantime, MINOS far detector data [25] and IceCube data [26] set tight limits on the $\mu-\tau$ oscillation channel SME coefficients. The last untested channel is the $e-\tau$ sector, and this can be tested by the reactor $\bar{v}_{e}$ disappearance data, because nonzero Lorentz violating neutrino oscillation in the $e-\tau$ channel would contribute to the reactor neutrino disappearance. We analyzed data from the Double Chooz reactor experiment, where $\sim 4 \mathrm{MeV}$ reactor $\bar{v}_{e}$ are detected by the detector located at $\sim 1050$ m away.

Figure 8 shows the result. Since the reactor power varies with a day-night cycle, the neutrino flux is a function of the solar time (period $86400.0 \mathrm{sec}$ ) and it can mimic the sidereal time variation effect (period 86164.1 sec), unless data taking is continuous in all one year. This is not the case for Double Chooz. However, the reactor cycle effect is simulated and taken into account in our analysis. We found that the data over simulation is flat and the data is consistent with no sidereal time dependence. Therefore we set limits on $e-\tau$ sector SME coefficients.

With the addition of this work, most of SME coefficients of all neutrino oscillation channels are constraint. Since neutrino oscillation is an interference experiment, as opposed to time of flight (TOF) which is a kinematic measurement, neutrino oscillation experiment is far more sensitive to small effect such as Lorentz violation. Therefore it is difficult to explain superluminal neutrinos observed by the OPERA experiment [28] while keeping all null Lorentz violation signals in 


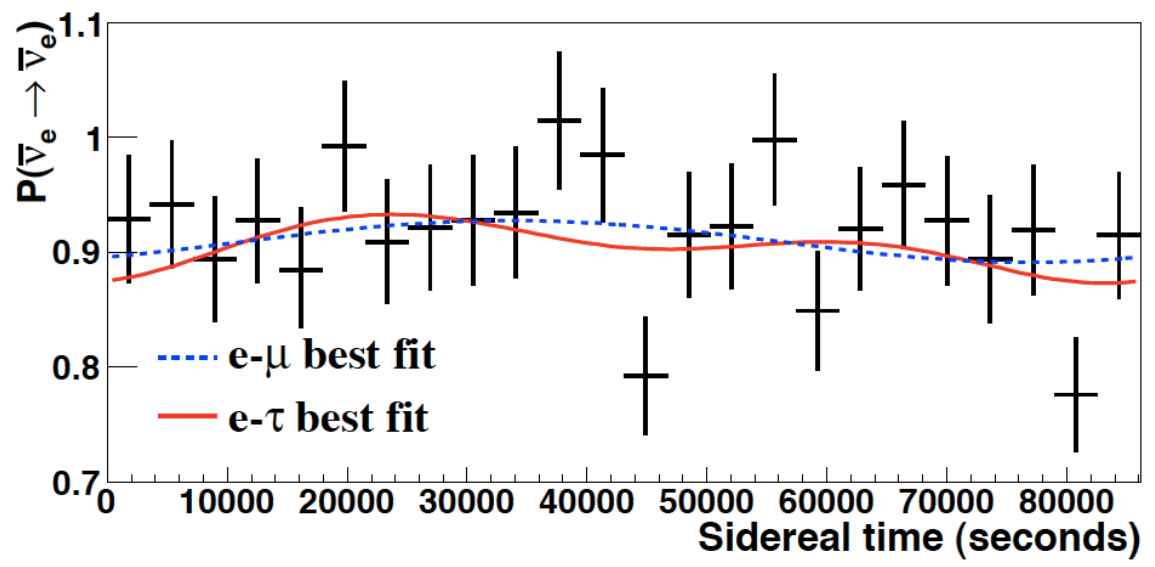

Figure 8: The fit results for the reactor antineutrino data at Double Chooz far detector. The ratio of data to simulation is overlaid with the best fit curves of models with Lorentz violation. Figure is taken from [27].

neutrino oscillation experiments. Therefore, it will be challenging to detect Lorentz violation in the neutrino sector in any terrestrial experiments. In the future, astrophysical neutrinos [29] may improve sensitivity to Lorentz violation by many orders of magnitude compared to these limits.

\section{Conclusions}

There is a world wide effort to test Lorentz violation with various state-of-art technologies, including neutrino oscillations. LSND and MiniBooNE data suggest Lorentz violation is an interesting solution to neutrino oscillations. MiniBooNE neutrino data prefer a sidereal time independent solution, and MiniBooNE antineutrino data prefer a sidereal time dependent solution, although statistical significance is not high. Limits from MiniBooNE exclude simple Lorentz violation motivated scenario for LSND. Finally, MiniBooNE, LSND, MINOS, IceCube, and Double Chooz set stringent limits on Lorentz violation in neutrino sector in terrestrial level.

\section{Acknowledgements}

I thank Jorge Díaz for valuable comments on this manuscript. I also thank to the ICHEP 2012 organizers and IUPAP C11 committee for the invitation to the ICHEP2012 conference.

\section{References}

[1] Teppei Katori, Mod. Phys. Lett. A 27, 1230024 (2012).

[2] For example, see, V. A. Kostelecký and S. Samuel, Phys. Rev. D 39, 683 (1989).

[3] V. A. Kostelecký and N. Russell, Rev. Mod. Phys. 83, 11 (2011).

[4] D. Colladay and V. A. Kostelecký, Phys. Rev. D 55, 6760 (1997); 58, 116002 (1998);

V. A. Kostelecký, Phys. Rev. D 69, 105009 (2004).

[5] L. B. Auerbach et al., Phys. Rev. D 72, 076004 (2005). 
[6] V. A. Kostelecký and M. Mewes, Phys. Rev. D 69, 016005 (2004).

[7] V. A. Kostelecký and M. Mewes, Phys. Rev. D 85, 096005 (2012).

[8] V. A. Kostelecký and M. Mewes, Phys. Rev. D 70, 076002 (2004).

[9] J. S. Díaz, V. A. Kostelecký, and M. Mewes, Phys. Rev. D 80, 076007 (2009).

[10] A. A. Aguilar et al., Phys. Rev. D 64, 112007 (2001).

[11] Teppei Katori, arXiv:1008.0906 [hep-ex].

[12] T. Katori, V. A. Kostelecký, and R. Tayloe, Phys. Rev. D 74, 105009 (2006).

[13] V. A. Kostelecký and M. Mewes, Phys. Rev. D 70, 031902 (R) (2004).

[14] J. S. Díaz and V. A. Kostelecký, Phys. Lett. B 700, 70025 (2011); Phys. Rev. D 85, 016013 (2012).

[15] Y. Abe et al., Phys. Rev. Lett. 108, 131801 (2012); F. P. An et al., Phys. Rev. Lett. 108, 171803 (2012); J. K. Ahn et al., Phys. Rev. Lett. 108, 191802 (2012).

[16] A. A. Aguilar-Arevalo et al., Phys. Rev. D 79, 072002 (2009).

[17] A. A. Aguilar-Arevalo et al., Nucl. Instrum. Meth. A 599, 28 (2009).

[18] R. B. Patterson et al., Nucl. Instrum. Meth. A 608, 206 (2009).

[19] A. A. Aguilar-Arevalo et al., Phys. Rev. Lett. 100, 032301 (2008).

[20] A. A. Aguilar-Arevalo et al., Phys. Rev. Lett. 102, 101802 (2009).

[21] A. A. Aguilar-Arevalo et al., Phys. Rev. Lett. 105, 181801 (2010).

[22] A. A. Aguilar-Arevalo et al., arXiv:1207.4809 [hep-ex].

[23] A. A. Aguilar-Arevalo et al., arXiv:1109.3480 [hep-ex].

[24] P. Adamson et al., Phys. Rev. Lett. 101, 151601 (2008); Phys. Rev. D 85, 031101 (2012).

[25] P. Adamson et al., Phys. Rev. Lett. 105, 151601 (2010).

[26] R. Abbasi et al., Phys. Rev. D 82, 112003 (2010).

[27] Y. Abe et al., arXiv:1209.5810 [hep-ex].

[28] T. Adam et al., JHEP 1210, 093 (2012).

[29] R. Abbasi et al., Nature, 484, 351 (2012). 